\title{
A Family of Hybrid Space-Time Codes for MIMO Wireless Communications
}

\author{
M. Bazdresch*1, J. Cortez ${ }^{2}$, O. Longoria-Gándara ${ }^{1}$, R. Parra-Michel ${ }^{3}$ \\ ${ }^{1}$ ITESO \\ Periférico Sur Manuel Gómez Morín 8585 \\ Tlaquepaque, Jalisco, México \\ *miguelb@ieee.org \\ 2 ITSON \\ Antonio Caso S/N, \\ C.P. 85130 , \\ Cd. Obregon, Sonora, México \\ ${ }^{3}$ CINVESTAV del IPN \\ Av. del Bosque 1145 \\ C.P. 45019 \\ Zapopan, Jalisco, México
}

\begin{abstract}
Hybrid MIMO space-time codes combine the benefits of spatial multiplexing with diversity gain to achieve both high spectral efficiency and link reliability. In this paper, we present a family of hybrid codes, known as LD STBC-VBLAST codes, along with a receiver architecture suitable for low-complexity hardware implementation. We show that, under Rayleigh fading, the performance of $\angle D S T B C-V B L A S T$ codes is superior to other recently proposed hybrid codes. We also present a technique to derive, from a given propagation scenario, spatially correlated MIMO channel models adequate for space-time coding performance analysis. Using this technique, we evaluate the performance of $L D$ STBC-VBLAST codes under several correlated channels.
\end{abstract}

Keywords: Linear dispersion codes, hybrid space-time codes, correlated MIMO channel, MIMO systems, sorted QR decomposition.

\section{RESUMEN}

Los códigos espacio-temporales híbridos para sistemas MIMO combinan las ventajas del multiplexaje espacial con la ganancia en diversidad, para lograr, al mismo tiempo, una alta eficiencia espectral y una baja tasa de error. En este artículo, presentamos una familia de códigos híbridos, conocida como códigos LD STBC-VBLAST, junto con una arquitectura para un receptor que puede ser implementada en hardware y presenta baja complejidad. Mostramos que, cuando el canal inalámbrico es Rayleigh, el rendimiento de los códigos LD STBC-VBLAST es mayor que el de otros códigos híbridos que han sido propuestos recientemente. También presentamos una técnica para derivar, a partir de un escenario de propagación, un canal MIMO con correlación espacial que es adecuado para simular y evaluar el rendimiento de un código espacio-temporal. Utilizando esta técnica, evaluamos el rendimiento de los códigos $L D S T B C-V B L A S T$ en varios canales correlacionados.

\section{Introduction}

A multiple-input multiple-output (MIMO) communications system uses $n_{T}$ antennas to transmit and $n_{R}$ antennas to receive. Provided that the wireless channel presents rich-scattering and independent Rayleigh fading between each pair of antennas, a MIMO system has the potential to achieve a higher data rate with lower probability of error, increasing neither bandwidth nor power, compared to single-antenna systems [1]. In this paper, we will present and analyze transmission schemes that are able to extract this potential extra performance.

Let $\mathrm{S}$ be a signal constellation with elements in $\mathbb{C}$, and $\mathbf{s}=\left[s_{1}, s_{2}, \ldots, s_{n_{T}}\right]^{T}$ a $n_{T} \times 1$ vector of symbols to be transmitted, where $s_{i} \in S, i=1,2,3, \ldots, n_{T}$ and where $(\cdot)^{T}$ is the transpose operator. Each symbol $s_{i}$ may be transmitted at certain time intervals, over 
certain antennas. A space-time block code (STBC) is a mapping of symbols to combinations of time intervals and antennas. This mapping may be represented by a table or a matrix, where each row corresponds to a transmit antenna and each column to a time interval. As an example, consider a system with $n_{T}=2$; the vector to be transmitted is $\mathbf{s}=\left[\begin{array}{ll}s_{1} & s_{2}\end{array}\right]^{T}$. An STBC may be specified by a matrix

$$
S=\left[\begin{array}{cc}
S_{1} & -s_{2}^{*} \\
S_{2} & s_{1}^{*}
\end{array}\right]
$$

which may be interpreted as follows: at the first symbol interval, antenna 1 transmits $s_{1}$ and antenna 2 transmits $s_{2}$. During the second interval, antenna 1 transmits $-s_{2}^{*}$ and antenna 2 transmits $s_{1}^{*}$, where $(\cdot)^{*}$ means complex conjugate. It is assumed that the channel remains constant during the two symbol intervals.

Compared to a SISO system, there are two ways an STBC may exploit the MIMO channel to obtain performance gains: diversity and spatial multiplexing. Assuming the channel between each pair of transmit and receive antennas is uncorrelated with the others, repeating transmission of a symbol over several antennas will provide spatial diversity. The maximum diversity of a MIMO channel is given by $n_{T} n_{R}$.

Spatial multiplexing, on the other hand, takes advantage of the fact that a MIMO system is equivalent to a system with $n_{\min }=\min \left(n_{R}, n_{T}\right)$ parallel, independent channels. Each of these channels may be used to transmit a different data stream, thereby increasing the data rate by a factor $n_{\text {min }}$ compared to a SISO system. In other words, the received signal has $n_{\min }$ complex dimensions (or $n_{\text {min }}$ complex degrees of freedom).

These two types of gains may appear to be mutually exclusive and, indeed, the first STBCs focused on exploiting either diversity or spatial multiplexing. In order to obtain maximum diversity, each symbol $s_{i}$ should be repeated over space and time as many times as possible, which precludes any rate gain. On the other hand, maximum data rate gain means repeating symbols as few times as possible, limiting the diversity gain.

In fact, it has been found that MIMO systems have a diversity-multiplexing tradeoff [2]. It is possible, in theory, to design STBCs where the data rate $r_{b}$ scales as

$$
r_{b}=r \log _{2}(S N R)
$$

and the error probability $p_{e}$ scales as

$$
p_{e} \approx S N R^{-d(r)} \text {. }
$$

Here, $r$ is the multiplexing gain and $d(r)$ is the diversity gain, which is given by

$$
d(r)=\left(n_{R}-r\right)\left(n_{T}-r\right),
$$

where $r$ takes values between 0 and $n_{\text {min }}$, and $d(r)$ takes values between 0 and $n_{T} n_{R}$. In practice, a given code may achieve gains that are lower than these optimal values. Such a code is said to achieve a suboptimal tradeoff. The most representative example of a code that achieves maximum data rate gain is V-BLAST [3]; the Alamouti scheme [4] is one of the best well-known maximum diversity gain codes.

How to design space-time codes that provide the desired gains while still being decodable with reasonable complexity? This is an area of ongoing research. We may divide the current efforts in two camps. On one hand, there are results based on information theory and algebraic number theory that concentrate on designing optimal codes with maximum $d(r)$ for all possible values of $r$; maximum-likelihood decoding at the receiver is assumed (for an overview of work in this area, see [5]). One of the stronger results obtained so far are the so-called perfect space-time codes, which meet a large number of design criteria that theoretically guarantee optimum performance. However, it has been proved that perfect STBCs exist only for a very limited range of $n_{T}$ and $n_{R}$ [6].

On the other hand, an alternative technique, known as hybrid coding, has emerged (see [7, 8] for early examples of hybrid codes). A hybrid space-time system tries to achieve desired gains $r$ and $d(r)$ by layering, in the transmitter, two or more space-time sub-systems, some of which provide diversity gain (for example, using Alamouti encoding) while the others provide data rate gain (using, for instance, V-BLAST encoding). More precisely, the $n_{T}$ transmit antennas are partitioned in disjoint sets, and each set is used to transmit a 
different data stream using a specific space-time code. This technique has the advantage of being very simple to design and offers significant flexibility in choosing an overall system data rate and performance.

When considering hybrid codes a natural question emerges: how can such a mixture of diversity and multiplexing codes be efficiently decoded? Several methods have been proposed; see [8, 9, 10, 11] for some examples. In [8], for instance, the receiver proceeds in stages. First, a spatial linear filter attempts to cancel the interference on one of the diversity layers, which was introduced by the remaining layers. This layer is then decoded using the maximum-likelihood criterion. Using the receiver's channel knowledge, the diversity-coded signal is regenerated and subtracted from the next layer, which is decoded in turn, until all layers are decoded. This algorithm presents high complexity and suffers from noise enhancement when attempting to cancel interference; its performance has been improved upon by more recent coding schemes [12].

In this work we present a less complex method, based on linear dispersion codes (LDCs) [13]. LDCs are a very general family of STBCs, allowing considerable flexibility in the way each information symbol is spread over time and space. In fact, well-known codes such as V-BLAST, Alamouti and Perfect STBCs are particular cases of LDCs. One key feature of LDCs is that they may be decoded using a technique known as ordered successive interference cancellation (OSIC), first introduced as a decoder for V-BLAST. This technique, while suboptimal, is well understood and feasible to implement. There is a link between hybrid codes and LDCs [8, 14], which allows hybrid codes to be expressed as equivalent LDCs and, as a consequence, allowing them to be decoded with OSIC. In general, the transformation from hybrid to purely spatial LDC results in a much larger channel matrix compared to the original hybrid code, which translates to increased receiver complexity. However, we show that the LDC channel matrix is highly structured, which may be used to achieve substantially lower complexity. Furthermore, receiver performance may be improved by detecting diversity-coded symbols before spatiallymultiplexed ones, as suggested in [8].
This paper is organized as follows. In Section 2, we will present a MIMO channel model (that may include correlation) that justifies the system model and assumptions made later. In Section 3, we introduce a hybrid STBC and present the transmitter and receiver architectures. In Section 4, we present in detail a low-complexity detection algorithm based on OSIC, whose performance (both on uncorrelated and correlated channels) is obtained and analyzed in Section 5. In the same section we show that the proposed receiver, when implemented in fixed-point hardware, exhibits excellent numerical stability and robustness. We present our conclusions in Section 6.

\section{Channel Model}

\subsection{MIMO Channel System}

In a MIMO system, $n_{T} n_{R}$ data streams propagate in a rich-scattering environment. The base-band model of the communication mechanism is given by

$$
\mathbf{r}=H \mathbf{s}+\mathbf{n},
$$

where transmitted vector $\mathbf{s}$ was defined above. In this model, $\mathrm{n}$ is an additive white circularly symmetric complex Gaussian noise (AWGN) vector with mean $E\{\mathbf{n}\}=0$ and covariance matrix $E\left\{\mathrm{nn}^{H}\right\}=N_{0} I_{n_{R}}$, where $E\{\cdot\}$ is the expectation operator, $(\cdot)^{H}$ denotes complex conjugate transpose, and $I_{n_{R}}$ is the $n_{R} \times n_{R}$ identity matrix. The received symbols are given by vector $\mathrm{r}=$ $\left[\begin{array}{llll}r_{1} & r_{2} & \ldots & r_{n_{R}}\end{array}\right]^{T}$, where $r_{i}$ (for $i=1,2, \ldots, n_{R}$ ) is the (complex) symbol received by antenna $i$. Matrix $H$ is the $n_{R} \times n_{T}$ channel matrix

$$
H=\left[\begin{array}{ccc}
h_{11} & \cdots & h_{n_{T}} \\
\vdots & \ddots & \vdots \\
h_{1 n_{R}} & \cdots & h_{n_{R} n_{T}}
\end{array}\right],
$$

where each element $h_{i j}$ represents the complex tap gain between transmit antenna $j$ and receive antenna $i$. Assuming slow, non-frequency selective fading, the channel matrix remains constant for the transmission of at least one (and possibly several) symbol vectors.

Under these assumptions, the spatial correlation function (SCF) sampled at the antennas' position 
results in the spatial correlation tensor (SCT), or full correlation matrix, given by

$$
R_{H}=E\left\{\operatorname{vec}(H) \operatorname{vec}(H)^{H}\right\},
$$

where the operator $\operatorname{vec}(\cdot)$ rearranges a matrix as a vector column-wise. Initial investigations [3] on this topic assume that the channel matrix elements are independent and identically distributed (i.i.d.) complex Gaussian random variables with zero mean and unit variance, so that the SCF is $R_{H}=I_{n_{R} n_{T}}$. Under this assumption, a MIMO system has large capacity [1]. However, in real propagation environments, the fades are not independent due to insufficient spacing of antennas and relative displacement between transmitter and receiver. This phenomenon is typically called spatial selectivity (SS), and it is said that the channel is under spatial correlation (SC) conditions.

It has been observed [15] that when the fades are correlated, channel capacity is significantly smaller than when fades are i.i.d. In the remainder of this section, we will describe the phenomenology behind the fading in correlated scenarios specifically considering SS (but not time or frequency selectivity).

When independence between transmitter and receiver is assumed (separability condition), the channel model can be characterized by partial information from the SCF via the Kronecker Model [16]:

$$
H=\left(R_{H}^{R X}\right)^{\frac{1}{2}} G\left(R_{H}^{T X}\right)^{\frac{H}{2}}
$$

where

$$
R_{H}^{T X(R X)}=\left(R_{H}^{T X(R X)}\right)^{\frac{1}{2}}\left(R_{H}^{T X(R X)}\right)^{\frac{H}{2}} .
$$

Matrix $G$ is an i.i.d. Gaussian random complex matrix whose elements have zero mean and variance equal to one, and $R_{H}^{T X(R X)}$ is the transmitted (received) spatial correlation matrix (SCM). The SCT and the SCM of transmitter and receiver are related by $R_{H}=R_{H}^{T X} \otimes R_{H}^{R X}$, where $\otimes$ denotes the Kronecker product.

\subsection{Power Azimuth Spectrum}

In order to evaluate the effects of SS on the performance of MIMO systems, it is necessary to develop a mechanism to generate channel realizations that is capable of accomplishing equation (7), for a given SCF (see [17] and references therein).

We will focus on Gaussian processes only. These processes are completely described by their second order statistics, that is, by their SCF. For this reason, it is sufficient to have the SCF to obtain suitable channel realizations. A Gaussian process' power distribution function may be understood intuitively since it depends on the spatial reference angles. It is a useful starting point for channel simulation. In Section 2.3 we will explain how to calculate the SCF starting from the distribution function.

Let us first describe the typical MIMO communication scenario where the transmitter and receiver sides are implemented with antenna arrays with a given topology. Here we focus on the uniform linear array (ULA) [17]; an example of this system is shown in Figure 1. As its name indicates, the antennas are arranged uniformly over a straight line. Spherical coordinates are used to indicate each antenna's position, using $\vartheta$ and $\varphi$ to denote the elevation and azimuthal angles, respectively. The position vector $\rho$ is given by

$$
\rho=[\cos (\varphi) \cos (\vartheta), \sin (\varphi) \cos (\vartheta), \sin (\vartheta)]^{T} .
$$

The wave vector of the departing/incident wave front in a far field scenario is expressed as

$$
\mathbf{k}=k_{0}[\cos (\varphi) \cos (\vartheta), \sin (\varphi) \cos (\vartheta), \sin (\vartheta)]^{T},
$$

where $\lambda$ is the wavelength of the carrier frequency and $k_{0}=2 \pi / \lambda$ is the free-space wave number of a single wavefront. From here on, we assume the array is oriented parallel to $y$ - axis (as depicted in Figure 1). 


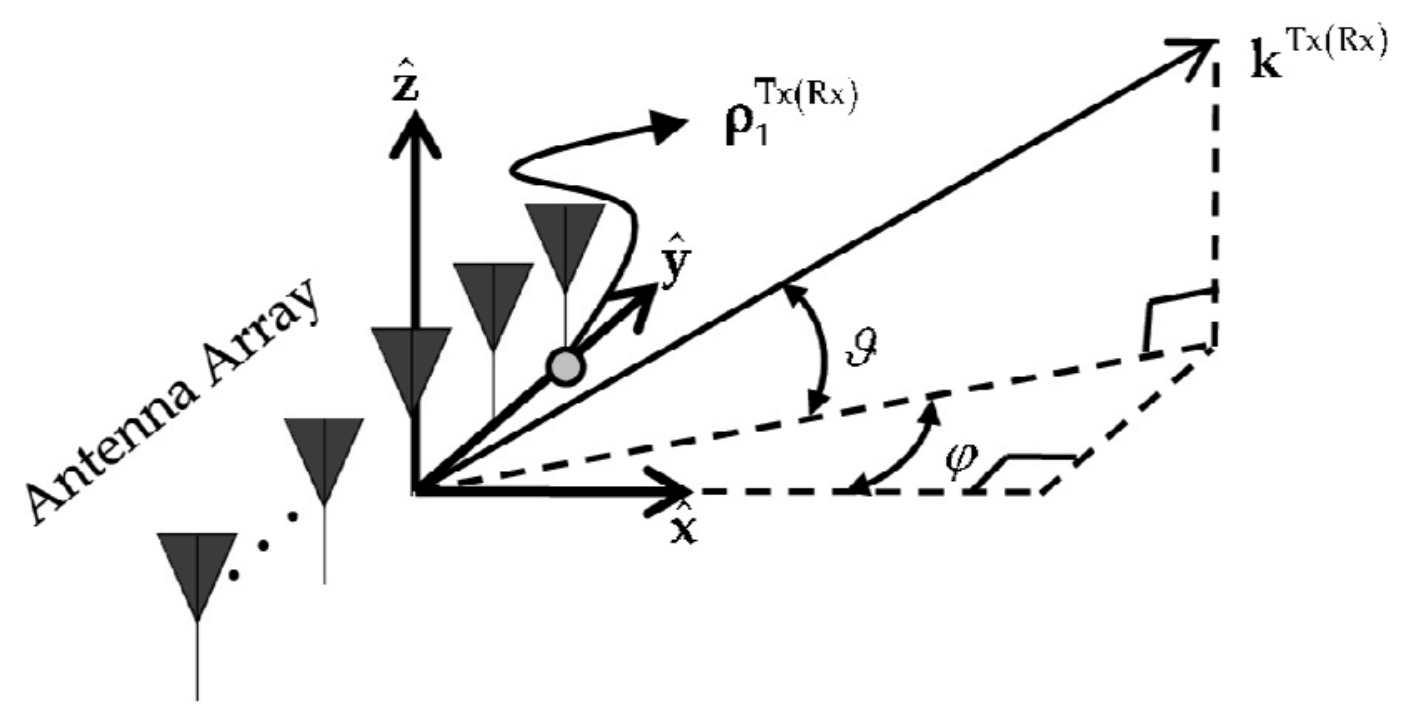

Figure 1. Representation of wave fronts as vectors in spherical coordinates.

A physical consequence of a SS scenario on signal behavior is that transmission may be done at different angles of departure (AoD) in elevation and azimuthal coordinates, and even the same signal can impact the receiver array at different angles of arrival (AoA). For practical purposes, only the azimuthal channel model is considered; that is, the direction of each wavefront is parallel to a given plane ( $x y$ in Figure 1); therefore, the elevation angle is assumed to be zero.

Under SS conditions, signal power is assumed to be a random variable that depends on AoA $\left(\varphi^{R x}\right)$ and $\operatorname{AoD}\left(\varphi^{T x}\right)$; its probability density function (PDF) is interpreted as a power distribution. For this reason, it is necessary to introduce the crosspower azimuth spectrum (xPAS), denoted by $p\left(\varphi^{R x}, \varphi^{T x}\right)$. This function describes the power distribution of the transmitted and received signals as a function of $\varphi^{R x}$ and $\varphi^{T x}$.

Under separability conditions it is useful to have a function that describes separately the power distribution at the transmitter and receiver; such a function is called power azimuth spectrum (PAS) and is denoted by $p\left(\varphi^{T x}\right)$ as the PAS in the transmitter, and by $p\left(\varphi^{R x}\right)$ as the PAS in the receiver. These functions satisfy $p\left(\varphi^{R x}, \varphi^{T x}\right)=$ $p\left(\varphi^{R x}\right) p\left(\varphi^{T x}\right)$ for the assumed Kronecker model.

Several standards and measurement campaigns have been developed to estimate the PAS at each side in different environments, such as the IEEE 802.15 standard proposed for wireless personal area networks (PAN) at $60 \mathrm{GHz}$; the METRA project [18], in macrocell and microcell environments; the COST 259 standard [19], for macrocell, urban and suburban environments. An important compilation of aperture angle parameters is presented in [20]. Table 1 summarizes the corresponding mathematical expressions for typical PAS considered in MIMO communication systems. All of them are centered at zero degrees.

An example of each cited PAS is depicted in Figure 2, which shows a uniform PDF with maximum aperture angle of $30^{\circ}$ and a truncated Gaussian PDF with maximum aperture angle of $90^{\circ}$ 


\begin{tabular}{|c|c|c|}
\hline PAS & Formula & Parameters \\
\hline Uniform & $p(\varphi)=\frac{1}{\varphi_{\max }-\varphi_{\min }},-\pi \leq \varphi_{\min } \leq \varphi \leq \varphi_{\max } \leq \pi$ & Interval $\left(\varphi_{\min }, \varphi_{\max }\right)$ \\
\hline Laplacian & $p(\varphi)=\frac{e^{-\frac{1}{k}|\varphi|}}{2 k\left(1-e^{-\frac{\pi}{k}}\right)},-\pi \leq \varphi \leq \pi$ & Decay Parameter $k$ \\
\hline Gaussian & $p(\varphi)=\frac{e^{-\frac{1}{2 \sigma^{2}} \varphi^{2}}}{\sqrt{2 \pi \sigma^{2}}},-\pi \leq \varphi \leq \pi$ & Variance $\sigma^{2}$ \\
\hline
\end{tabular}

Table 1. Power azimuth spectrum (PAS) functions frequently used to model physical environments.
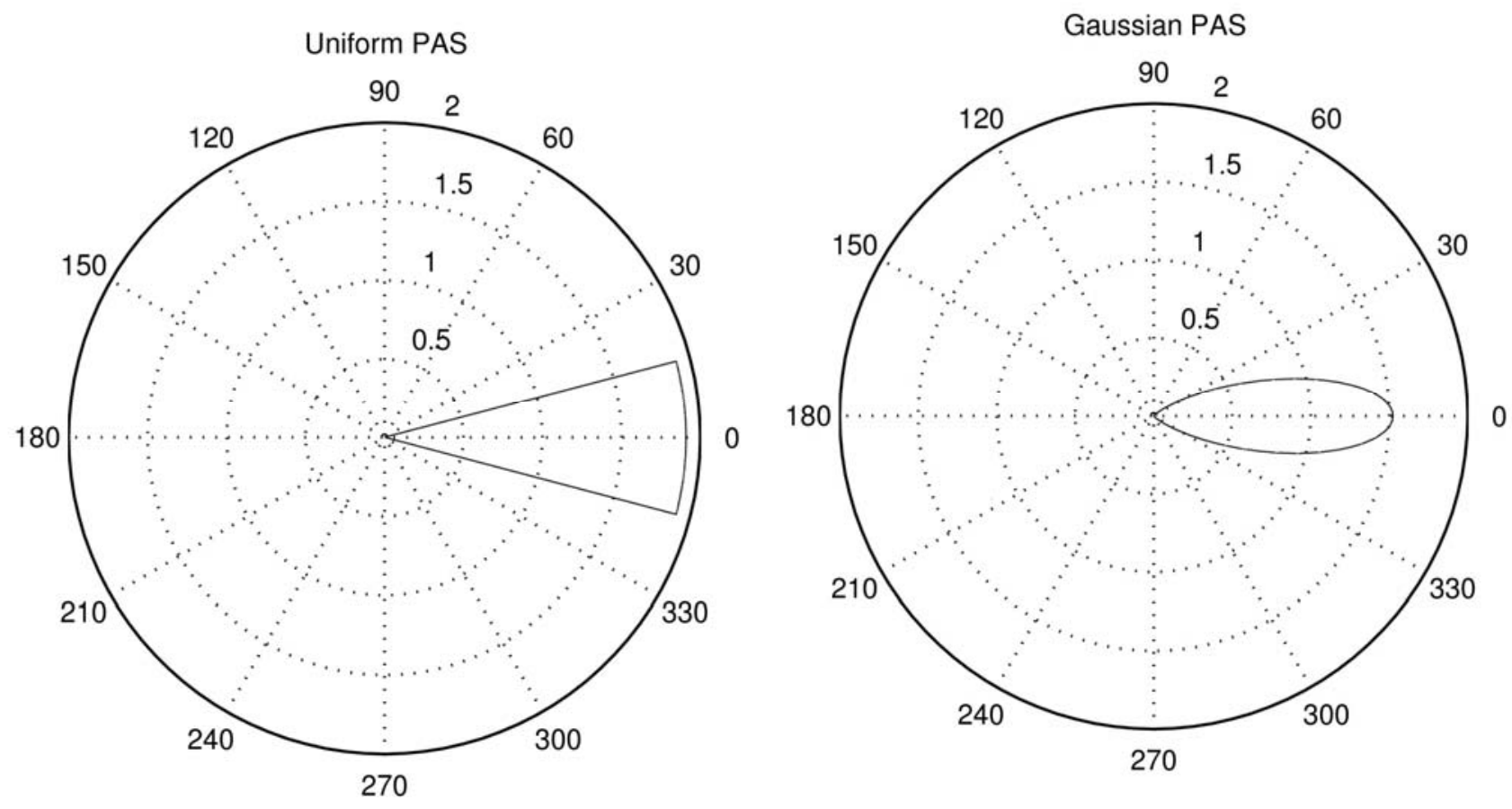

Figure 2. Polar coordinate plots of power azimuth spectra. a) Uniform b) Gaussian. 


\subsection{PAS and the SCF}

As explained above, the main goal of channel characterization is to devise a mechanism capable of generating channel realizations that follow the model established in Equation (8); for this purpose the SCM is required. However, most measurement campaigns provide second order statistics data only for the PAS. A practical mathematical tool that relates $p\left(\varphi^{T x(R x)}\right)$ to $R_{H}^{T x(R x)}$ at the transmitter (or receiver), employed in [17], uses the well-known array manifold vector (AMV), which is given by

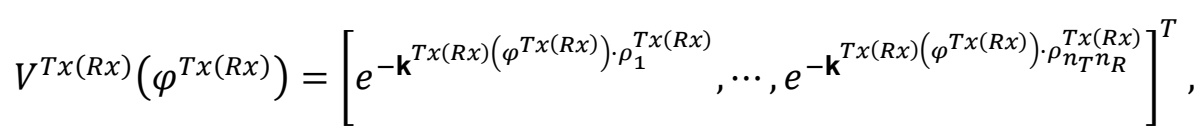

where $\mathbf{k}^{T x(R x)}$ is the wave vector at the transmitter (or receiver); $\varphi^{T x(R x)}$ is the AoD (or AoA); $\rho_{m(n)}^{T x(R)}$ is the position vector of the $m$ th (or $n$ th) antenna (given a designated order) at the transmitter (or receiver), $m=1,2, \ldots, n_{T}$ (or $n=1,2, \ldots, n_{R}$ ), and $n_{T}$ (or $n_{R}$ ) is the number of antennas at the transmitter (or receiver).

For an ULA, the AMV is reduced to

$$
V^{T x(R x)}\left(\varphi^{T x(R x)}\right)=\left[e^{\frac{-2 \pi j}{\lambda} d_{1}^{T x(R x)} \sin \left(\varphi^{T x(R x)}\right)}, \cdots, e^{\frac{-2 \pi j}{\lambda} d_{n_{T} n_{R}}^{T x(R x)} \sin \left(\varphi^{T x(R x)}\right)}\right]^{T},
$$

where $d_{k}^{T x(R x)}$ represents the linear position of the $k$ th antenna at the transmitter (or receiver), and $\lambda$ is the signal wavelength. In Equation (13), the array is oriented as in Figure 1.

The direct formula to calculate a SCM is

$$
R_{H}^{T x(R x)}=\int_{\varphi^{T x(R x)}} p\left(\varphi^{T x(R x)}\right) V^{T x(R x)}\left(\varphi^{T x(R x)}\right) V^{T x(R x)}\left(\varphi^{T x(R x)}\right)^{H} d \varphi^{T x(R x)} .
$$

Once the transmitter and receiver's spatial correlation matrices are known, it is possible to calculate the channel realizations needed for the desired performance test.

\section{Hybrid Code Description}

We now present a hybrid code based on linear dispersion codes [13] that may be used to transmit over MIMO channels as have been modeled in the previous section. We call this code LD STBC-VBLAST [21]. We present the space-time mapping in the transmitter, and the receiver's architecture.

\subsection{Space-Time Mapping}

This hybrid code partitions the transmit antennas into $n_{L}=n_{S}+n_{B}$ sets, called spatial layers. Layers 1 to $n_{S}$ operate as V-BLAST, transmitting one symbol per time period without repetition. Each of the $n_{B}$ layers is a 2-antenna Alamouti encoder. A single data stream is converted to $n_{L}$ parallel streams, each of which is mapped to a given constellation and then transmitted on one of the spatial layers. The total number of transmit antennas is $n_{T}=n_{S}+2 n_{B}$. The system block diagram is shown in Figure 3 . 
More specifically, a block of symbols $s_{1}, s_{2}, \ldots, s_{n_{s y m}}$, where $n_{\text {sym }}=2\left(n_{S}+n_{B}\right)$, is mapped to each antenna as specified in Table 2; on average, $\left(2 n_{S}+n_{B}\right)$ symbols are transmitted per channel use. The code rate, then, is $\frac{n_{S}+n_{B}}{2 n_{T}}$. Note that the rate may be varied by changing the number and spatial layering distribution of the transmit antennas. In general, the rate will be higher than a (quasi-)orthogonal code's, but less than that obtained with V-BLAST for the same number of transmit antennas. The power allocated to the V-BLAST layers is given by:

$$
P_{S}=\frac{2}{n_{\text {sym }}}
$$

while the power allocated to the Alamouti encoders is given by

$$
P_{A}=\frac{P_{S}}{2} \text {. }
$$

This allocation results in all symbols being transmitted with the same energy, which is the optimal strategy in the absence of channel state information (CSI) at the transmitter.

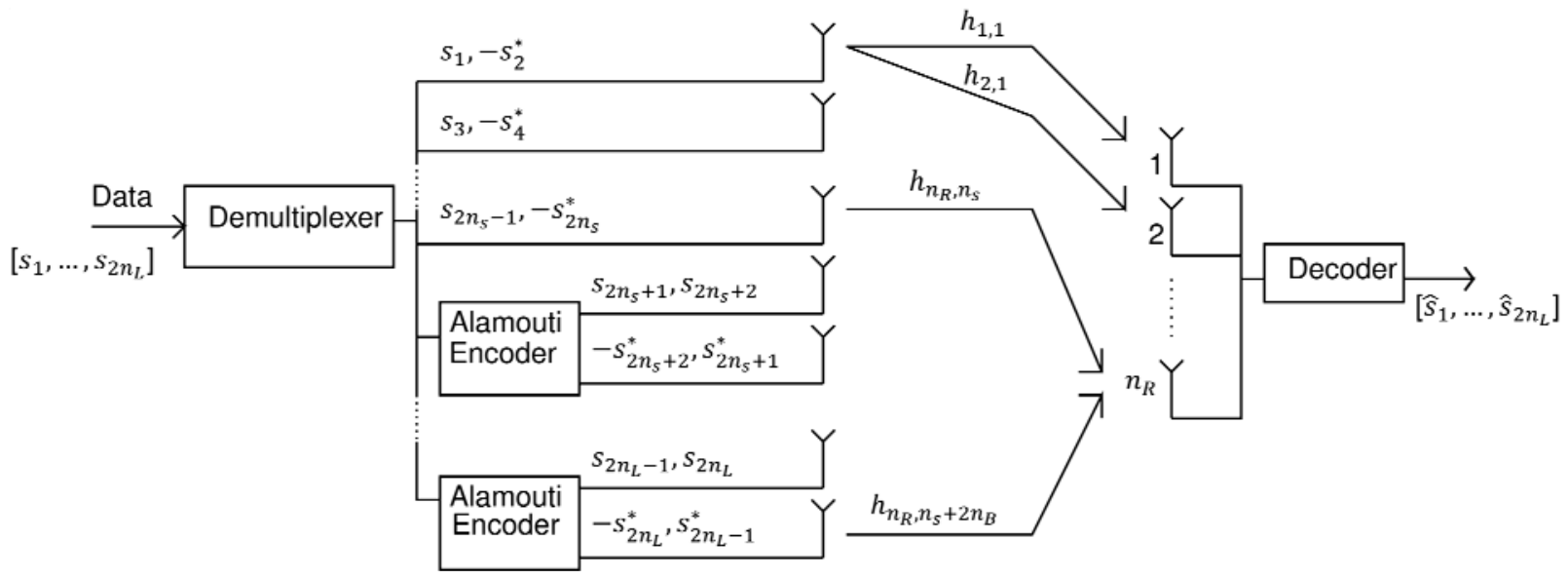

Figure 3. LD STBC-VBLAST transceiver architecture. A vector of symbols $\left[s_{1}, \ldots, s_{2 n_{L}}\right]$ is transmitted over two symbol periods; the first $2 n_{S}$ are V-BLAST encoded and the rest are Alamouti encoded. The channel between transmit antenna j and receive antenna

\begin{tabular}{|c|c|c|c|c|c|c|c|}
\hline \multicolumn{3}{|c|}{ Spatial Antennas } & \multicolumn{5}{|c|}{ STBC Antennas } \\
\hline 1 & $\cdots \quad V$ & $n_{S}$ & $\begin{array}{c}\text { Block } 1 \\
\left(k=2 n_{S}+1\right) \\
1\end{array}$ & $\begin{array}{r}\text { Bloc } \\
\left(k=2 n_{S}\right. \\
1\end{array}$ & $\begin{array}{c}\cdots \\
2 B-1) \\
2\end{array}$ & $\begin{array}{r}\text { Block } n_{B} \\
\left(k=2 n_{S}+\right. \\
1\end{array}$ & $\left.n_{B}-1\right)$ \\
\hline$s_{1}$ & $s_{2 V-1}$ & $s_{2 n_{S}-1}$ & $s_{k+1}$ & $s_{k}$ & $s_{k+1}$ & $s_{k}$ & $s_{k+1}$ \\
\hline$-s_{2}^{*}$ & $-s_{2 V}^{*}$ & $-s_{2 n_{S}}^{*}$ & $-s_{k+1}^{*} \quad s_{k}^{*}$ & $-s_{k+1}^{*}$ & $s_{k}^{*}$ & $-s_{k+1}^{*}$ & $s_{k}^{*}$ \\
\hline
\end{tabular}
$i$ has gain $h_{i j}$, which is shown for some antenna pairs.

Table 2. LD STBC-VBLAST symbol to antenna mapping, for two consecutive symbol periods. 


\subsection{Receiver Architecture}

We assume $n_{R} \geq n_{S}+n_{B}$ and perfect CSI at the receiver. The transmitted signal may be written as $S=\left[\begin{array}{ll}S_{\text {spa }} & S_{A}\end{array}\right]^{T}$, where $S_{\text {spa }}$ corresponds to the symbols transmitted by V-BLAST layers and $S_{A}$ to the symbols transmitted by the Alamouti encoders, so that

$$
S_{s p a}=\left[\begin{array}{ll}
s_{1} & -s_{2}^{*} \\
s_{3} & -s_{4}^{*} \\
\vdots & \vdots \\
s_{2 n_{S}-1} & -s_{2 n_{S}}^{*}
\end{array}\right]=\left[\begin{array}{cc}
s_{1}^{(1)} & s_{1}^{(2)} \\
s_{2}^{(1)} & s_{2}^{(2)} \\
\vdots & \vdots \\
s_{n_{S}}^{(1)} & s_{n_{S}}^{(2)}
\end{array}\right],
$$

and

$$
S_{A}=\left[\begin{array}{c}
S_{1}^{A} \\
S_{2}^{A} \\
\vdots \\
S_{n_{B}}^{A}
\end{array}\right]=\left[\begin{array}{cc}
S_{1}^{(1) A} & S_{1}^{(2) A} \\
\vdots & \vdots \\
S_{n_{B}}^{(1) A} & S_{n_{B}}^{(2) A}
\end{array}\right]
$$

where each element of (18) is given by

$$
\left[\begin{array}{ll}
S_{B}^{(1) A} & S_{B}^{(2) A}
\end{array}\right]=\left[\begin{array}{lr}
S_{k} & -S_{k+1}^{*} \\
S_{k+1} & S_{k}^{*}
\end{array}\right]
$$

with $B=1,2, \ldots, n_{B}$ and $k=2\left(n_{S}+B\right)-1$. Note that we have performed the mapping described in Table 2. In this notation, the super-index indicates time interval and the sub-index indicates spatial layer number. An $A$ in the super-index indicates an Alamouti layer.

It is assumed that the channel remains constant during the transmission of $S$, so that the received signal may be written as

$$
\left[\begin{array}{cc}
y_{1}^{(1)} & y_{1}^{(2)} \\
y_{2}^{(1)} & y_{2}^{(2)} \\
\vdots & \vdots \\
y_{n_{R}}^{(1)} & y_{n_{R}}^{(2)}
\end{array}\right]=\left[\begin{array}{ccc}
h_{11} & \cdots & h_{n_{T}} \\
\vdots & \ddots & \vdots \\
h_{1 n_{R}} & \cdots & h_{n_{R} n_{T}}
\end{array}\right]\left[\begin{array}{cc}
S_{S p a} \\
S_{A}
\end{array}\right]+\left[\begin{array}{cc}
n_{1}^{(1)} & n_{1}^{(2)} \\
n_{2}^{(1)} & n_{2}^{(2)} \\
\vdots & \vdots \\
n_{n_{R}}^{(1)} & n_{n_{R}}^{(2)}
\end{array}\right],
$$

or, in matrix form,

$$
Y=H S+N,
$$

where $Y \in \mathbb{C}^{n_{R} \times 2}$ represents the symbols received in a block, $H \in \mathbb{C}^{n_{R} \times n_{T}}$ is the channel matrix, and $N \in \mathbb{C}^{n_{R} \times 2}$ is the Gaussian noise added to each received symbol.

Reformulating the system Equation (20) as a linear dispersion code, we have:

$$
\left[\begin{array}{c}
y_{1}^{(1)} \\
y_{1}^{(2)^{*}} \\
\vdots \\
y_{n_{R}}^{(1)} \\
y_{n_{R}}^{(2)^{*}}
\end{array}\right]=\left[\begin{array}{ll}
H_{s p a} & H_{A}
\end{array}\right] S_{L D}\left[\begin{array}{c}
n_{1}^{(1)} \\
n_{1}^{(2)^{*}} \\
\vdots \\
n_{n_{R}}^{(1)} \\
n_{n_{R}}^{(2)^{*}}
\end{array}\right],
$$

which can be expressed as a matrix equation:

$$
Y_{L D}=H_{L D} S_{L D}+N_{L D},
$$

where block matrix $H_{L D}=\left[\begin{array}{ll}H_{s p a} & H_{A}\end{array}\right] \in \mathbb{C}^{2 n_{R} \times 2 n_{L}}$ is called a linear dispersion matrix. One block of $H_{L D}$ corresponds to the V-BLAST layers, and the other to the Alamouti layers. The V-BLAST block $H_{\text {spa }}$ is given by

$$
H_{s p a}=\left[\begin{array}{cccc}
H_{11}^{s p a} & H_{12}^{s p a} & \cdots & H_{1 n_{S}}^{s p a} \\
H_{21}^{s p a} & H_{22}^{s p a} & \cdots & H_{2 n_{S}}^{s p a} \\
\vdots & \vdots & \ddots & \vdots \\
H_{n_{R} 1}^{s p a} & H_{n_{R} 2}^{s p a} & \cdots & H_{n_{R} n_{S}}^{s p a}
\end{array}\right]
$$

Where

$$
H_{i j}^{s p a}=\left[\begin{array}{cc}
h_{i j} & 0 \\
0 & -h_{i j}^{*}
\end{array}\right]
$$

for $i=1,2, \ldots, n_{R}$ and $j=1,2, \ldots, n_{S}$. The Alamouti block $H_{A}$ is itself a block matrix; it is given by

$$
H_{A}=\left[\begin{array}{cccc}
H_{11}^{A} & H_{12}^{A} & \cdots & H_{1 n_{B}}^{A} \\
H_{21}^{A} & H_{22}^{A} & \cdots & H_{2 n_{B}}^{A} \\
\vdots & \vdots & \ddots & \vdots \\
H_{n_{R} 1}^{A} & H_{n_{R}}^{A} & \cdots & H_{n_{R} n_{B}}^{A}
\end{array}\right],
$$




$$
H_{k B}^{A}=\left[\begin{array}{cc}
h_{k, 2 B+n_{S}-1} & h_{k, 2 B+n_{S}} \\
h_{k, 2 B+n_{S}}^{*} & -h_{k, 2 B+n_{S}-1}^{*}
\end{array}\right]
$$

for $k=1,2, \ldots, n_{R}$ and $B=1,2, \ldots, n_{B}$. The matrix $H_{i j}^{s p a}$ is the portion of $H_{L D}$ that links the $j^{\text {th }}$ spatial antenna with the $i^{\text {th }}$ receiver antenna. Likewise, $H_{k B}^{A}$ links the $B^{t h}$ Alamouti block to the $k^{\text {th }}$ receiver antenna. To complete the reformulation of system Equation (21), we need to rearrange matrix $S$. We define $S_{L D} \in \mathbb{C}^{\left(2 n_{S}+n_{B}\right) \times 1}$

$$
S_{L D}=\left[\begin{array}{c}
S_{L D}^{s p a} \\
S_{L D}^{A}
\end{array}\right]
$$

where $S_{L D}^{s p a} \in \mathbb{C}^{2 n_{S} \times 1}$ and $S_{L D}^{A} \in \mathbb{C}^{n_{B} \times 1}$ are given by

$$
S_{L D}=\left[\begin{array}{c}
S_{L D}^{s p a} \\
S_{L D}^{A}
\end{array}\right]
$$

and

$$
S_{L D}^{A}=\left[S_{1}^{(1) A} S_{2}^{(1) A}, \cdots, S_{n_{B}}^{(1) A}\right]
$$

The reformulation of Equation (20) as (22) allows us to consider the hybrid MIMO system as a simpler, equivalent, purely spatial system with $N_{T}=2\left(n_{S}+n_{B}\right)$ transmit antennas and without distinction between the Alamouti and V-BLAST layers. This simpler system is shown in Figure 4. We are now ready to propose a receiver algorithm, based on the sorted-QR decomposition (SQRD) [22] and OSIC linear detection that takes advantage of the structure of the linear dispersion matrices to achieve low complexity and high performance.

\section{Detection Algorithm}

\subsection{OSIC Detection for LD STBC-VBLAST Hybrid Code}

We first find the sorted $Q R$ decomposition $Q_{L D} R_{L D}=H_{L D}$, where $Q_{L D}$ is unitary and $R_{L D}$ is upper triangular. By multiplying the received signal $Y_{L D}$ by $Q_{L D}^{H}$, we obtain a new received vector

$$
\tilde{Y}_{L D}=Q_{L D}^{H} Y_{L D}=R_{L D} S_{L D}+\widetilde{N}_{L D}
$$

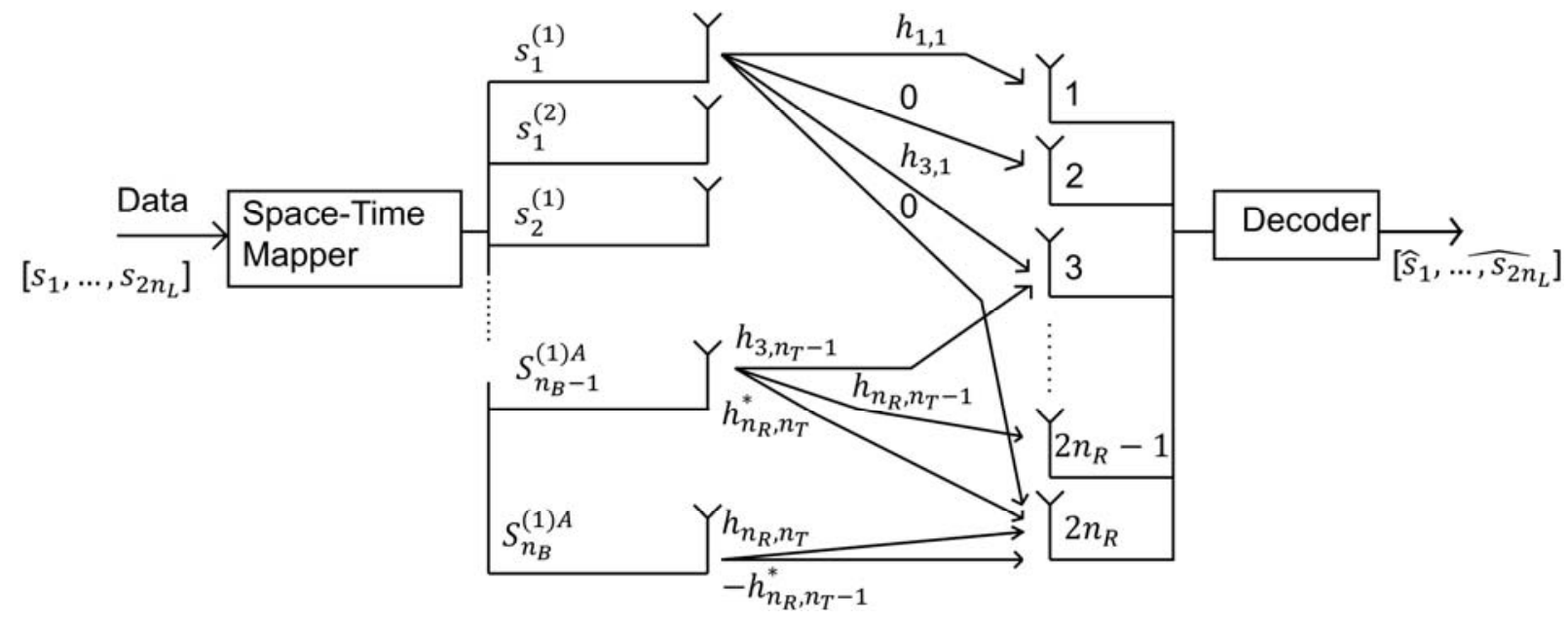

Figure 4. The $L D$ STBC-VBLAST hybrid code presented in Figure 3, transformed into a purely spatial linear dispersion code. The channel gains are given in matrix $H_{L D}$. 
Note that the statistical properties of the noise term $\widetilde{N}_{L D}$ are the same as $N$. Due to the upper triangular structure of $R_{L D}$, the $j^{\text {th }}$ element of $\tilde{Y}_{L D}$ is given by

$$
\tilde{y}_{j}=r_{j j} s_{j}+\sum_{i=j+1}^{N_{T}} r_{j i} \cdot s_{i}+\tilde{n}_{j} .
$$

The symbols are estimated in sequence, from lower stream to higher stream, using OSIC. Assuming that all previous decisions are correct, the interference can be perfectly canceled at each step except for the noise. The estimated symbol $\hat{s}_{j}$ is given by

$$
\hat{s}_{j}=D\left[\frac{\tilde{y}_{j}-\sum_{i=j+1}^{N_{T}} r_{j i} \cdot \hat{s}_{i}}{r_{j j}}\right],
$$

where $\hat{s}_{j}$ is the estimate of $s_{j}$ and $D[\cdot]$ is a decision device that maps its argument to the closest constellation point. The SQRD may be obtained using Givens rotations using an algorithm we call hybrid coding (HC) sorted QR decomposition or HC-SQRD.

\subsection{HC Sorted QR Decomposition}

Note that matrix $H_{L D} \in \mathbb{C}^{2 n_{R} \times N_{T}}$. A direct application of Givens rotations to calculate QR decomposition on it would result in very high complexity. This complexity may be reduced substantially by taking advantage of the matrix's structure. At the same time, we will implement Givens rotations using the coordinate rotational digital computer (CORDIC) algorithm [23], to allow an eventual fixed-point hardware implementation.

From (24), (25), (26), and (27) we can see that many of the elements of $H_{L D}$ are equal, and their locations in each matrix are fixed and can be calculated in advance. The method proposed involves obtaining the QR decomposition of $H_{L D}$ in two stages: first we obtain the $Q R$ decomposition corresponding to the spatial layers of the hybrid system; in the second stage we calculate the QR decomposition for the diversity layers. A block diagram of the process is shown in Figure 5.

In the first step, we calculate the sorted $Q R$ decomposition of matrix $H_{m}$, defined as

$$
H_{m}=\left[\begin{array}{cccccc}
h_{1,1} & h_{1,2} & \cdots & h_{1, n_{T}} & y_{1,1} & y_{1,2} \\
h_{2,1} & h_{2,2} & \cdots & h_{2, n_{T}} & y_{2,1} & y_{2,2} \\
\vdots & \vdots & \ddots & \vdots & \vdots & \vdots \\
h_{n_{R}, 1} & h_{n_{R}, 2} & \cdots & h_{n_{R}, n_{T}} & y_{n_{R}, 1} & y_{n_{R}, 2}
\end{array}\right] .
$$

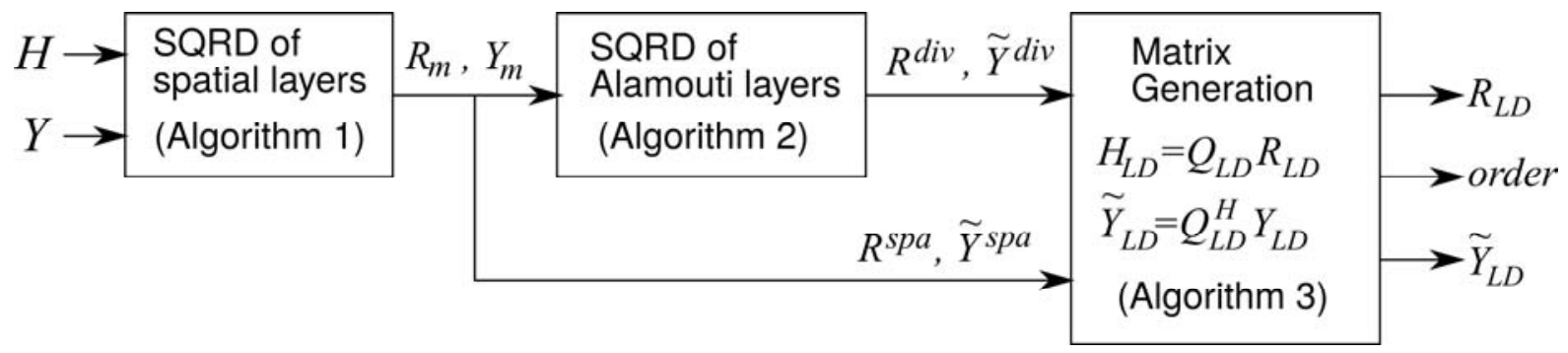

Figure 5. HC Sorted QR process using Givens rotations. Each block performs the indicated algorithm. 
We apply a series of orthogonal transformations $\Theta$ using Givens rotations on $H_{m}$ to obtain matrix $\bar{H}_{m}$ :

$$
\bar{H}_{m}=\left[\begin{array}{ccccccccc}
r_{1,1} & r_{1,2} & \cdots & r_{1, n_{S}} & r_{1, k} & \cdots & r_{1, n_{T}} & \tilde{y}_{1,1} & \tilde{y}_{1,2} \\
0 & r_{2,2} & \cdots & r_{2, n_{S}} & r_{2, k} & \cdots & r_{2, n_{T}} & \tilde{y}_{2,1} & \tilde{y}_{2,2} \\
\vdots & \vdots & \ddots & \vdots & \vdots & \ddots & \vdots & \vdots & \vdots \\
0 & 0 & \cdots & r_{n_{S}, n_{S}} & r_{n_{S}, k} & \cdots & r_{n_{S}, n_{T}} & \tilde{y}_{n_{S}, 1} & \tilde{y}_{n_{S}, 2} \\
0 & 0 & \cdots & 0 & \bar{h}_{k, k} & \cdots & \bar{h}_{k, n_{T}} & \bar{y}_{k, 1} & \bar{y}_{k, 2} \\
0 & 0 & \cdots & 0 & \bar{h}_{k+1, k} & \cdots & \bar{h}_{k+1, n_{T}} & \bar{y}_{k+1,1} & \bar{y}_{k 2} \\
\vdots & \vdots & \ddots & \vdots & \vdots & \ddots & \vdots & \vdots & \vdots \\
0 & 0 & \cdots & 0 & \bar{h}_{n_{R}, k} & \cdots & \bar{h}_{n_{R}, n_{T}} & \bar{y}_{n_{R}, 1} & \bar{y}_{n_{R}, 2}
\end{array}\right],
$$

where $k=n_{S}+1$. In this process, we also produce a vector order which specifies the detection order of the spatial layers. The operations performed on $H_{m}$ are detailed in Algorithm 1, where CORDIC operations are denoted as follows. Let $Z=a+j b$ be a complex number. The CORDIC vectorial operation is denoted $\left[X_{r} \Theta\right]=\mathbf{V}(a, b)$, and the CORDIC rotation is denoted $\left[X_{r} X_{i}\right]=$ $\mathbf{R}(a, b, \theta)$.

Next, we select the first $n_{S}$ rows of $\bar{H}_{m}$ to build the matrices $R^{\text {spa }}$ and $\tilde{Y}^{\text {spa }}$ :

$$
\begin{aligned}
R^{s p a} & =\left[\begin{array}{cccc}
r_{1,1} & r_{1,2} & \cdots & r_{1, n_{T}} \\
0 & r_{2,2} & \cdots & r_{2, n_{T}} \\
\vdots & \vdots & \ddots & \vdots \\
0 & 0 & \cdots & r_{n_{S}, n_{T}}
\end{array}\right], \\
\tilde{Y}^{s p a} & =\left[\begin{array}{cc}
\tilde{y}_{1,1} & \tilde{y}_{1,2} \\
\tilde{y}_{2,1} & \tilde{y}_{2,2} \\
\vdots & \vdots \\
\tilde{y}_{n_{S}, 1} & \tilde{y}_{n_{S}, 2}
\end{array}\right] .
\end{aligned}
$$

Matrices $R^{s p a}$ and $\tilde{Y}^{s p a}$ represent the contribution of the spatial layers in the hybrid scheme. The next step is building matrix $H_{\text {div }}^{\text {ala }}$ for the Alamouti layers, from elements $\bar{h}_{i, j}$ in (35), as follows (assuming $k=n_{S}+1 k=n_{S}+1$

$H_{\text {div }}^{\text {ala }}=\left[\begin{array}{ccccccc}\bar{h}_{k, k} & \bar{h}_{k, k+1}^{*} & \cdots & \bar{h}_{k, n_{T}-1} & \bar{h}_{k, n_{T}}^{*} & \tilde{y}_{k, 1} & \tilde{y}_{k, 2} \\ \bar{h}_{k+1, k} & \bar{h}_{k+1, k+1}^{*} & \cdots & \bar{h}_{k+1, n_{T}-1} & \bar{h}_{k+1, n_{T}}^{*} & \tilde{y}_{k+1,1} & \tilde{y}_{k+1,2} \\ \vdots & \vdots & \ddots & \vdots & \vdots & \vdots & \vdots \\ \bar{h}_{n_{R}, k} & \bar{h}_{n_{R}, k+1}^{*} & \cdots & \bar{h}_{n_{R}, n_{T}-1} & \bar{h}_{n_{R}, n_{T}}^{*} & \tilde{y}_{n_{R}, 1} & \tilde{y}_{n_{R}, 2}\end{array}\right]$

The SQRD of this matrix may be carried out using Algorithm 2. Finally, matrices $R_{L D}$ and $\tilde{Y}_{L D}$ are generated from matrices $R^{s p a}, R^{d i v}, \tilde{Y}^{s p a}$ and $\tilde{Y}^{d i v}$. The construction process is described in Algorithm 3. Once $R_{L D}$ and $\tilde{Y}_{L D}$ are calculated, symbol detection may be carried out according to the procedure described in Section 4.1. 


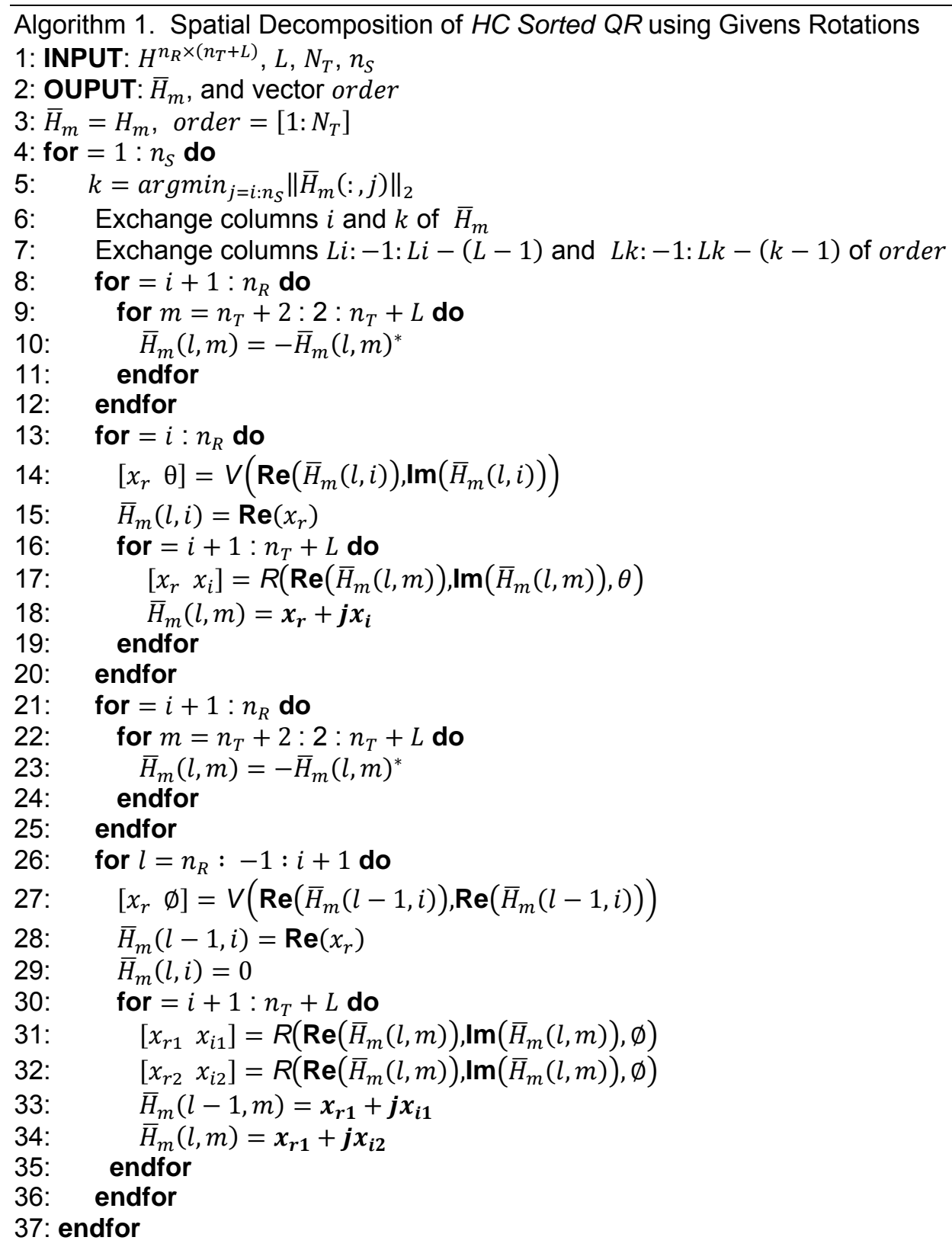




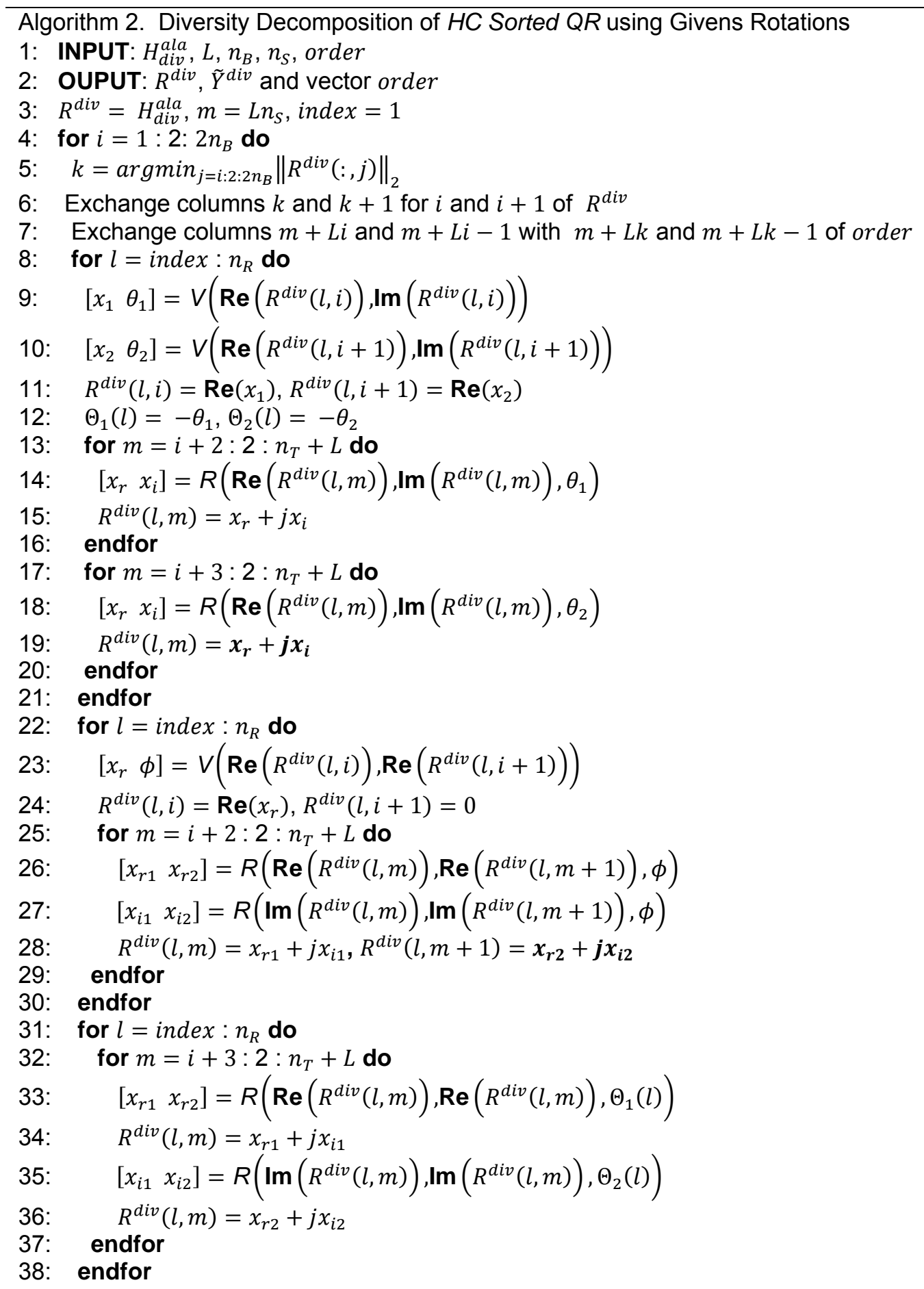




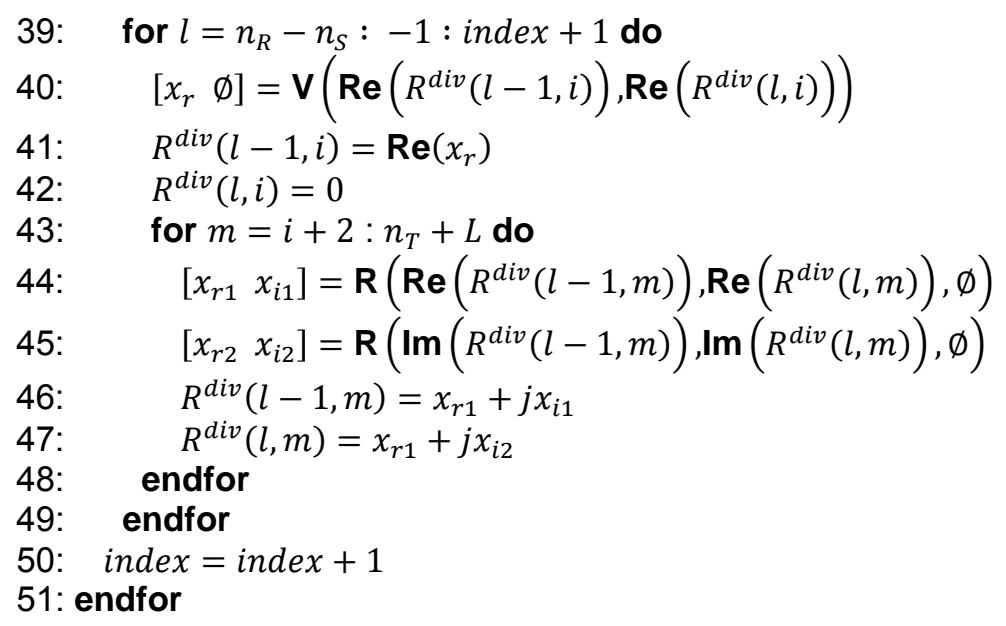

52: $\tilde{Y}^{d i v}=\left[\begin{array}{cc}R^{d i v}\left(1, n_{T}+1\right) & R^{d i v}\left(1, n_{T}+2\right) \\ R^{d i v}\left(2, n_{T}+1\right) & R^{d i v}\left(2, n_{T}+2\right) \\ \vdots & \vdots \\ R^{d i v}\left(n_{B}, n_{T}+1\right) & R^{d i v}\left(n_{B}, n_{T}+2\right)\end{array}\right]$

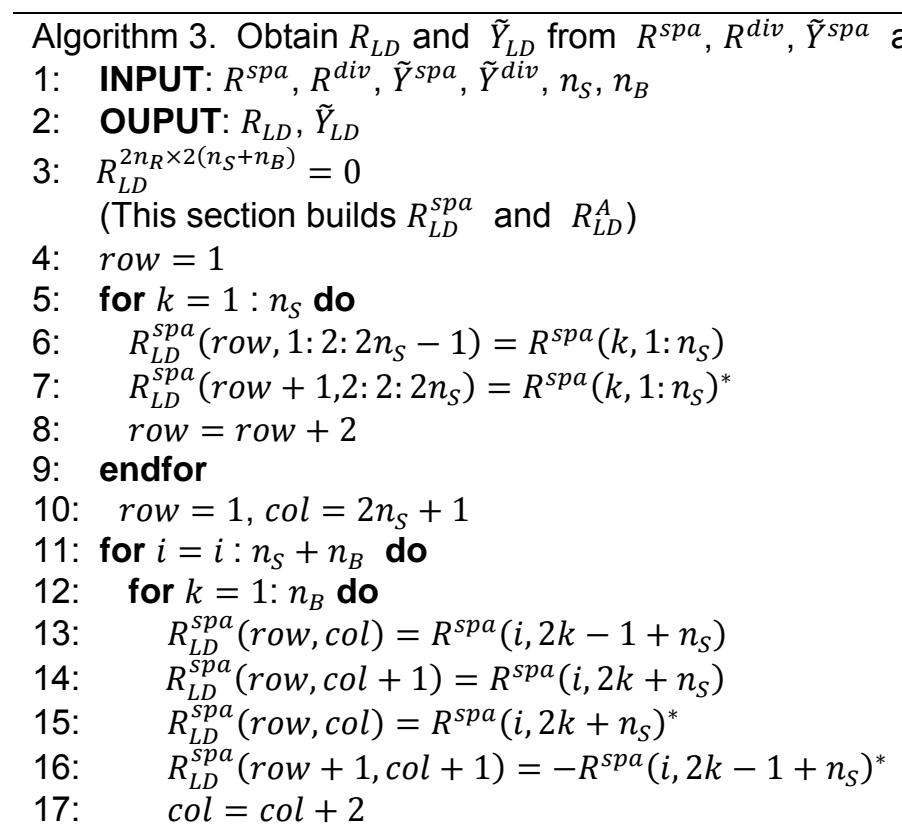




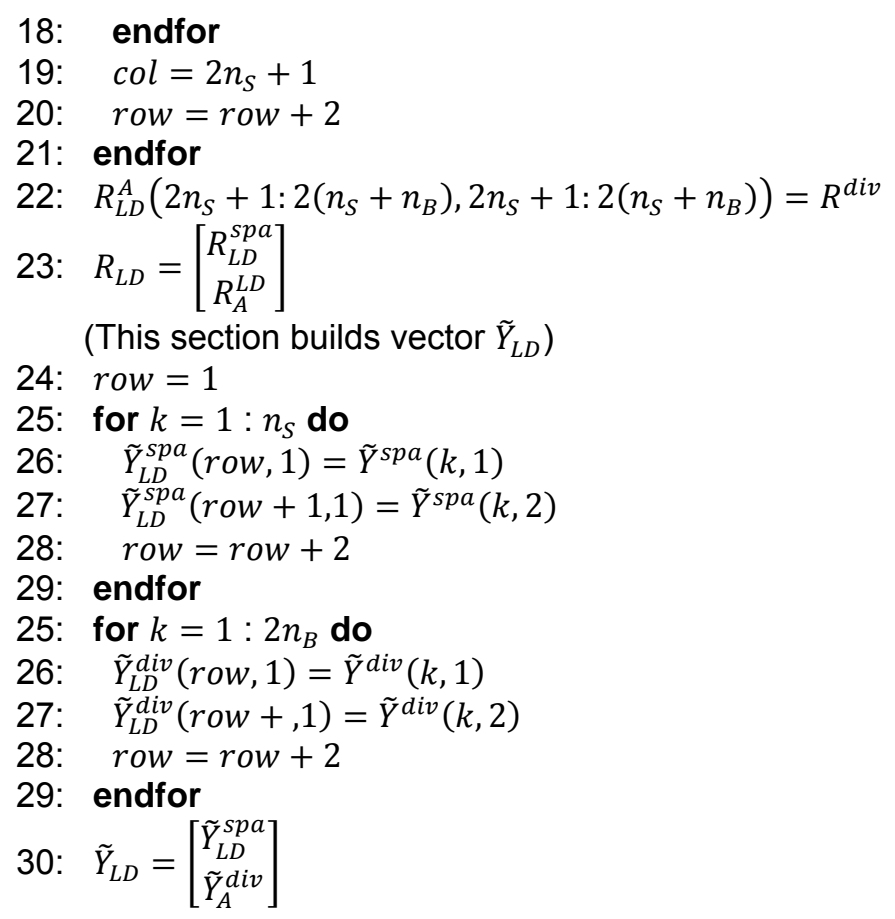

\section{Performance Analysis}

\subsection{Performance on Uncorrelated Channels}

In this section we compare the bit-error rate (BER) versus average signal-to-noise ratio (SNR) performance of $L D$ STBC-VBLAST, STBCVBLAST [10], QR Group Receiver [11], and Precoded STBC-VBLAST [24]. In all cases we assume 16-QAM modulation and a block length $L=2$. Simulations are run until 400 block errors are found. The code rate has been fixed to 3 symbols (12 bits) per channel use.

In Figure 6 (a), we compare the BER performance of QR Group Receiver $6 \times 6$, STBC-VBLAST $6 \times 6(2,2,3)$, and $L D S T B C-V B L A S T$ with $n_{R}=6$, $n_{T}=6$ and $n_{B}=3$. The $H C$ Sorted $Q R$ matrix has been calculated using double-precision floating point arithmetic and the modified Gram-Schmidt algorithm. With $n_{R}=6, n_{T}=6$ and $n_{B}=3$, at BER $=10^{-3}, L D$ STBC-VBLAST performs substantially better than the other codes.
In Figure 6 (b), we compare $L D S T B C-V B L A S T$ with PRECODED STBC-VBLAST. This hybrid code requires CSI at the transmitter, which is used to optimally allocate antenna power. With 3 symbols per channel use, this code requires $6 \times 6$ antennas. Without CSI and the extra cost of precoding, LD STBC-VBLAST with 6 antennas per side presents a coding gain of $2 \mathrm{~dB}$ over the precoded system, for $\mathrm{BER}=$ $10^{-4}$.

As previously mentioned, the $H C$ Sorted $Q R$ matrix may be calculated using the CORDIC algorithm. In Figure 7 we compare the modified Gram-Schmidt (MGS) algorithm with CORDIC, using 5, 6 and 7 iterations for a $6 \times 6$ system with 3 Alamouti encoders in the transmitter. It may be concluded that 7 iterations suffice to achieve BER performance just a fraction of a dB worse than MGS with double precision. 


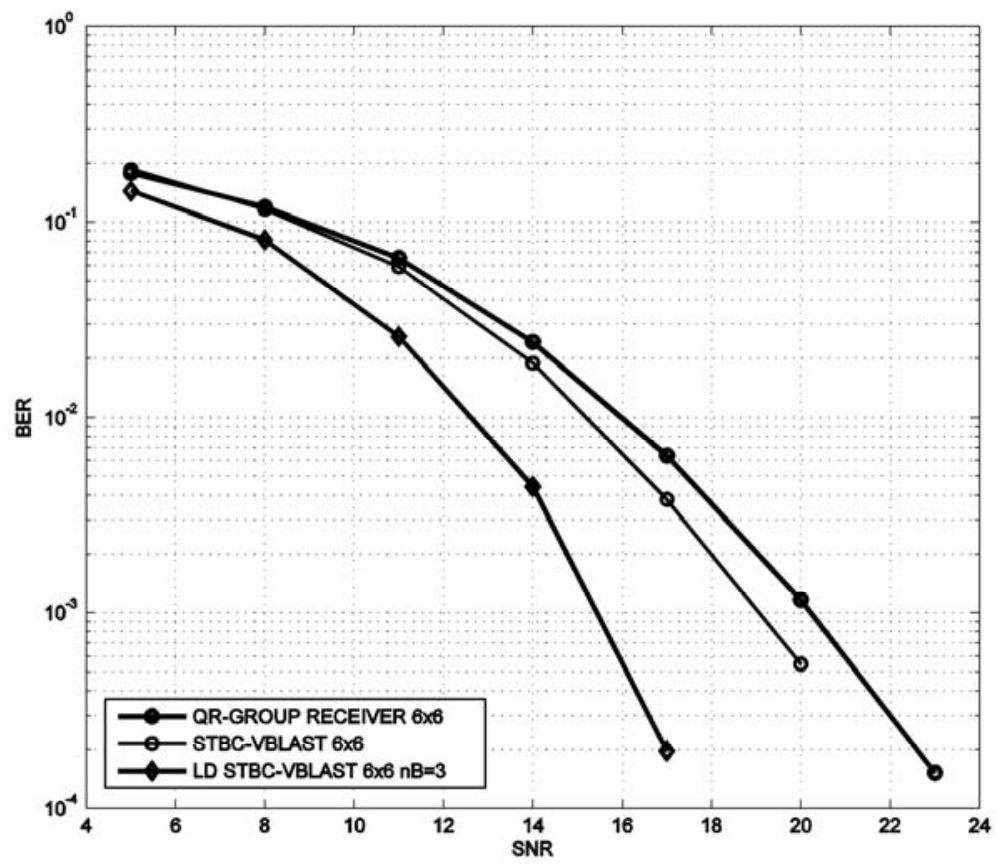

a)

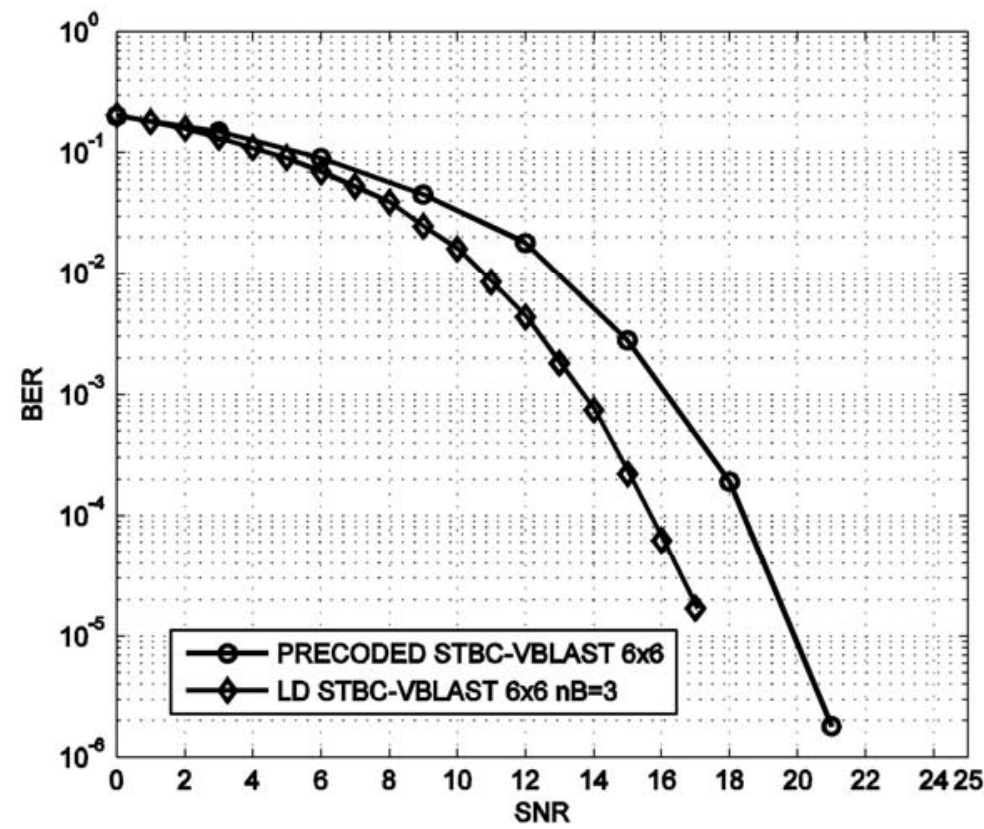

b)

Figure 6. BER vs. average SNR comparison.

a) $\angle D S T B C-V B L A S T$, STBC-VBLAST $6 \times 6(2,2,3)$, and QR Group Receiver $6 \times 6$.

b) $\angle D S T B C-V B L A S T$ and PRECODED STBC-VBLAST. 


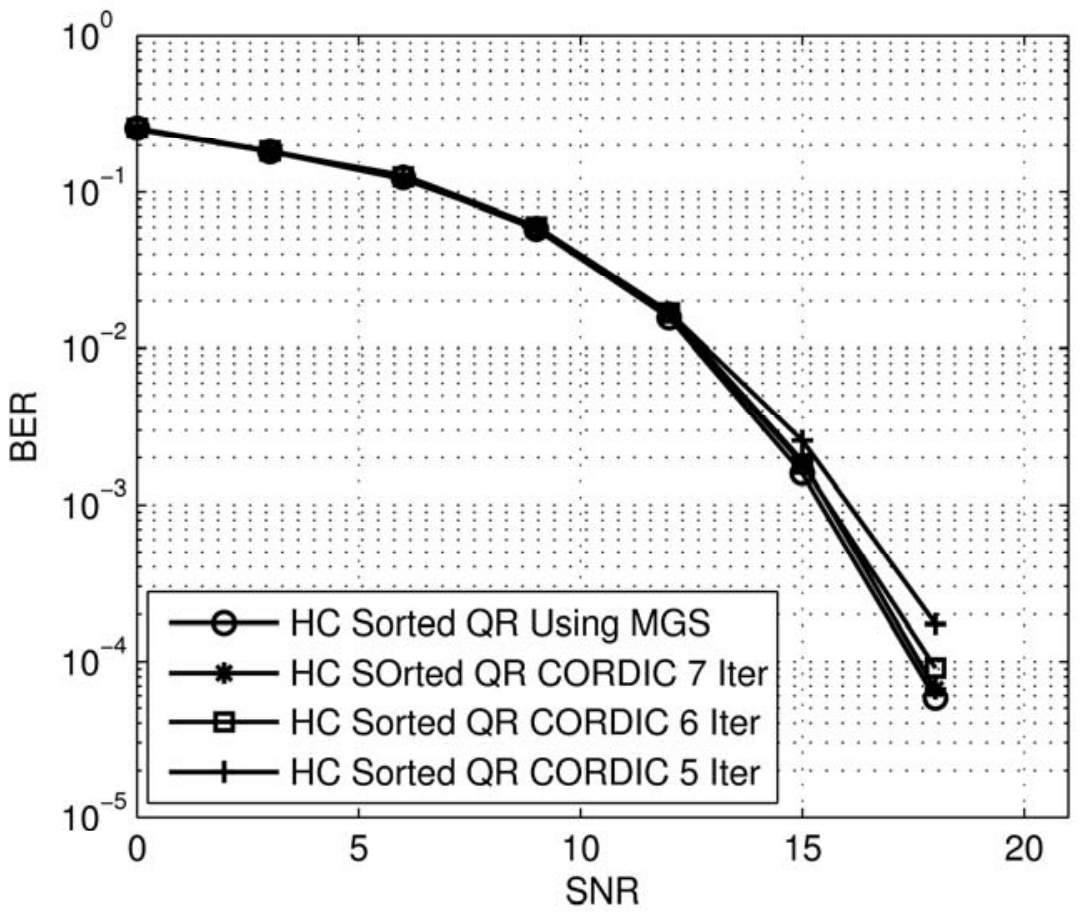

Figure 7. BER vs. SNR of $\angle D S T B C-V B L A S T 6 \times 6, n_{B}=3$ using CORDIC algorithm.

\subsection{Performance on Correlated Channels}

So far it has been assumed that the channel matrix has i.i.d. elements. A more realistic situation is that there is some degree of correlation present, which results in a loss of diversity and performance. To illustrate this loss, we introduce channel correlation in the following way: we assume a 90 degree transmit angle, 30 degree receive angle, and three different correlation distributions: Uniform, Laplacian, and Gaussian. In Figure 8, the performance loss is clearly visible, with Laplacian correlation the worst case (a loss of nearly $8 \mathrm{~dB}$ at $\mathrm{BER}=10^{-3}$ ).
The results presented above assume the $H C$ Sorted QR matrix has been calculated with double precision arithmetic. It is interesting to see the effect that correlation has on the feasibility of a practical implementation using CORDIC. As seen in Figure 8, the number of CORDIC iterations required to get results comparable to MGS has increased from 7 to 9 . Correlation, then, not only has an adverse effect on the receiver's BER performance, but it also requires a more complex hardware receiver as well. These results correspond to Laplacian distribution with the same angles as before. The receiver complexity increase is explained by the magnitude of the diagonal elements of matrix $R_{L D}$. Correlation results in smaller values, a good approximation of which requires more CORDIC iterations. 


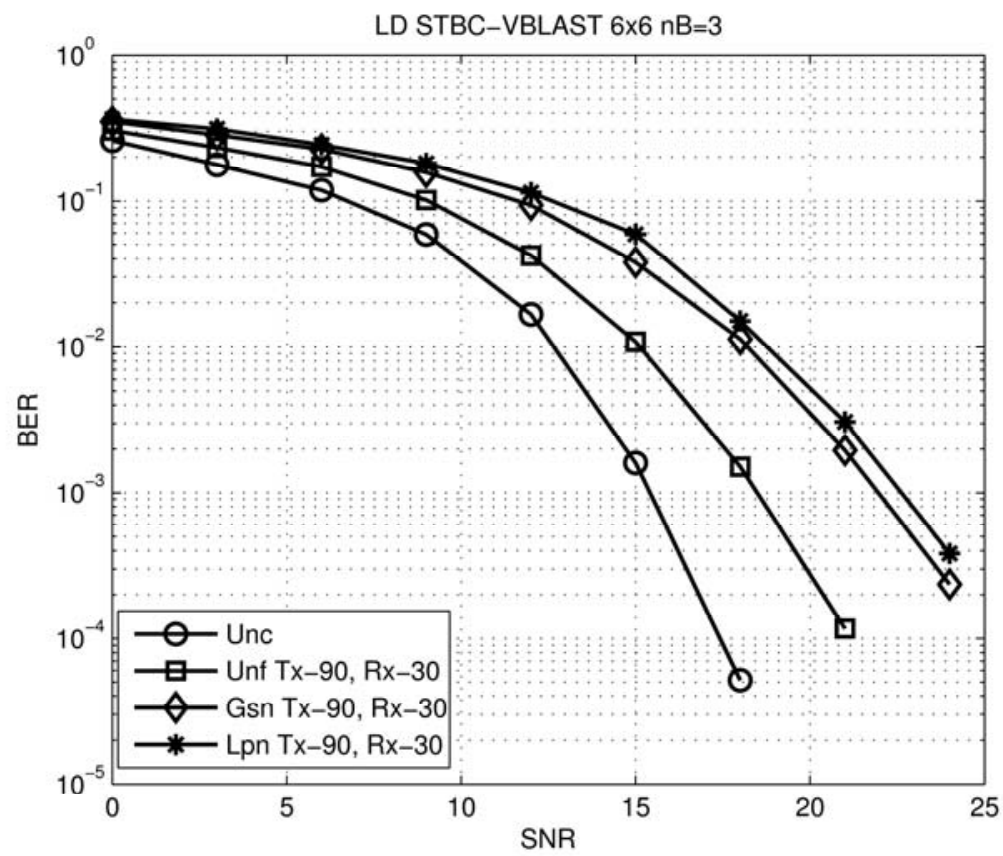

a)

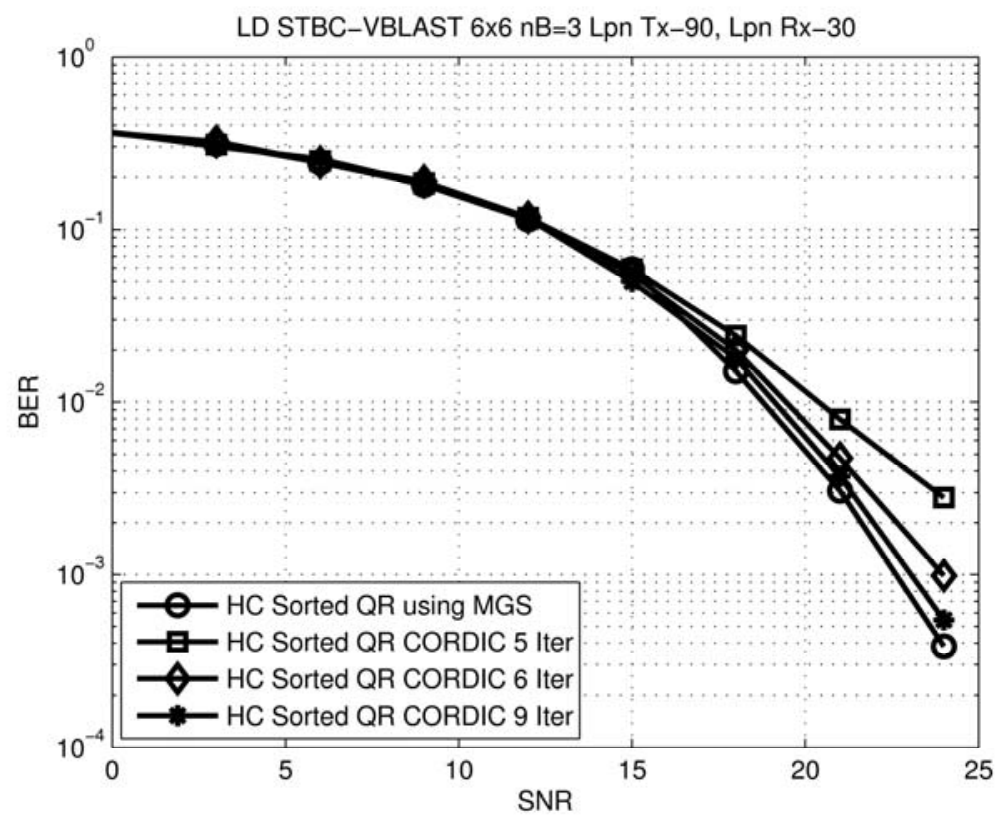

b)

Figure 8. LD STBC-VBLAST performance on correlated channels.

a) BER vs. SNR of LD STBC-VBLAST $6 \times 6, n_{B}=3$ on a correlated channel with 90 degree transmit angle and 30 degree receive angle. Unc is uncorrelated channel (included for reference), Unf is uniformly distributed correlation, Gsn is Gaussian, and Lpn is Laplacian.

b) BER vs. SNR of LD STBC-VBLAST $6 \times 6, n_{B}=3$, using CORDIC algorithm and correlated (Laplacian) channel. 


\section{Conclusions}

We have presented the $\angle D S T B C-V B L A S T$ family of hybrid space-time codes, along with a receiver algorithm with very low complexity. This receiver, based on the theory of linear dispersion codes, may be implemented in hardware with CORDIC using Givens rotations. We have shown that the BER performance of this family of codes is better than that of other hybrid codes that have been proposed recently. We have presented a methodology to generate correlated MIMO channel realizations with desired power azimuth spectrum, which we have used to evaluate the effect of correlation on LD STBC-VBLAST codes, on their $B E R$ performance as well as on the required receiver complexity increase.

\section{Acknowledgements}

This work was supported by PROMEP under research grant 103.5/10/8573.

\section{References}

[1] İ. Telatar, "Capacity of Multiple-Antenna Gaussian Channels", European Transactions on Telecommunications, vol. 10, no. 6, pp. 585-595, 1999.

[2] D. Tse and L.Zheng, "Diversity and Multiplexing: Fundamental Tradeoff in Multiple Antenna Channels", IEEE Transactions on Information Theory, Vol. 49, pp. 1073-96, 2003.

[3] G. J. Foschini, "Layered Space-Time Architecture for Wireless Communication in a Fading Environment When Using Multi-Element Antennas", Bell Laboratories Technical Journal, Vol. 1, No. 2, pp. 41-59, 1996.

[4] S. M. Alamouti, "A Simple Transmit Diversity Technique for Wireless Communications, IEEE Journal on Selected Areas in Communications", Vol. 16, No. 8, pp. 1451-1458, 1998.

[5] F. Oggier, J. C. Belfiore and E. Viterbo, "Cyclic Division Algebras: A Tool for Space-Time Coding", Foundations and Trends in Communications and Information Theory, Vol. 4, No. 1, pp. 1-95,2007.
[6] G. Berhuy and F. Oggier, "On the Existence of Perfect Space-Time Codes", IEEE Transactions on Information Theory, vol.55, No.5 , pp. 2078-2082, 2009.

[7] E.N. Onggosanusi, A.G. Dabak and T.A. Schmidl, "High rate space-time block coded scheme: performance and improvement in correlated fading channels", IEEE Proceedings of the Wireless Communications and Networking Conference (WCNC 2002), Vol.1, pp. 194199, March 2002.

[8] W. da C. Freitas Jr., F. R. P. Cavalcanti, and R. R. Lopes, "Hybrid Transceiver Schemes for Spatial Multiplexing and Diversity in MIMO Systems," Journal of Communication and Information Systems, vol. 20, no. 3, pp. 63-76, 2005.

[9] H. Kim, H. Park, T. Kim and I. Eo, "Performance Analysis of a DSTTD System with Decision-Feedback Detection", IEEE Proceedings of the International Conference on Acoustics, Speech and Signal Processing (ICASSP 2006), Vol. 4, pp. 749-752, May 2006.

[10] T. Mao and M. Motani, "STBC-VBLAST for MIMO Wireless Communication Systems", Proceedings of the IEEE International Conference on Communications (ICC 2005), vol. 4, pp. 2266-2270, May 2005.

[11] L. Zhao and V. K. Dubey, "Detection Schemes for Space-time Block Code and Spatial Multiplexing Combined System", IEEE Communication Letters, Vol. 9, No. 1, pp. 49-51, 2005.

[12] J. Cortez, M. Bazdresch, D. Torres, R. Parra-Michel, "Generalized ABBA-V-BLAST Hybrid Space-Time Code for Wireless Communications", IEEE 9th Workshop on Signal Processing Advances in Wireless Communications, 2008 (SPAWC 2008), pp. 481-485, 6-9 July 2008.

[13] B. Hassibi and B. M. Hochwald, "High-rate Codes That Are Linear in Space and Time", IEEE Transactions on Information Theory, Vol. 48, No. 7, pp. 1804-1824, 2002.

[14] O. Longoria-Gandara, A. Sanchez Hernandez, J. Cortez, M. Bazdresch and R. Parra, "Linear Dispersion Codes Generation from Hybrid STBC-VBLAST Architectures", IEEE Proceedings of the Fourth International Conference on Electrical and Electronics Engineering (ICEEE 2007), pp. 142-145, September 2007. 
[15] D. Gesbert, H. Bolcskei, D.A. Gore and J. Paulraj, "Outdoor MIMO Wireless Channels: Models and Performance Prediction", IEEE Transactions on Communications, vol. 50, no. 12, pp. 1926-1934, 2002.

[16] D. S. Shiu, G. J. Foshini, M. J. Gans and J. M. Kahn, "Fading Correlation and Its Effect on the Capacity of Multielement Antenna Systems", IEEE Transactions on Communications, Vol. 48, No. 3, pp. 502-512, 2000.

[17] R. Parra-Michel, A. Alcocer-Ochoa, A. SanchezHernandez and V. Kontorovich, " MIMO Channel Modeling and Simulation", in Recent Advances in Signal Processing, I-Tech Education and Publishing KG, ISBN 978-953-7619-41-1.

[18] J.R. Fonollosa, R. Gaspa, X. Mestre, A. Pages, M. Heikkila, J.P. Kermoal, L. Schumacher, A. Pollard and J. Ylitalo, "The IST METRA Project", IEEE Communications Magazine, Vol. 40, No. 7, pp. 78-86, 2002.

[19] F. Molish, H. Asplund, R. Heddergott, M. Steinbauer and T. Zwick, "The COST259 Directional Channel Model - Part I: Overview and Methodology", IEEE Transactions on Wireless Communications, Vol. 5, No. 12, pp. 34213433, 2006.

[20] S. Sesia, I. Toufik and M. Baker, "LTE: The UMTS Long Term Evolution, From Theory to Practice", John Wiley \& Sons Ltd, West Sussex, UK, 2009.

[21] J. Cortez, M. Bazdresch and A. Garcia, "Low Complexity Detector for STBC-VBLAST Architecture Based on SQRD Decomposition", IEEE Proceedings of 20th Personal, Indoor and Mobile Radio Communications Symposium (PIMRC 2009), September 2009, pp. 25492553.

[22] D. Wubben, R. Bohnke, J. Rinas, V. Kuhn and K.D. Kammeyer, "Efficient algorithm for decoding layered space-time codes", Electronics Letters, Vol. 37, No. 22, pp. 1348-1350, 2001.

[23] J. E. Volder, "The CORDIC trigonometric computing technique", IRE Transactions on Electronic Computers, vol. EC-8, pp.330 -334 1959

[24] C. Meng and J. Tuqan, "PRECODED STBCVBLAST for MIMO Wireless Communication Systems", IEEE Proceedings of the International Conference on Acoustics, Speech and Signal Processing (ICASSP 2007), Vol. 3, pp. 337-340, May 2007. 